\title{
Knockdown of PNO1 inhibits esophageal cancer progression
}

\author{
GUOWEN WANG ${ }^{1}$, QICAI LI $^{1}$, CHUANKUI LI ${ }^{1}$, GUIXIN DUAN ${ }^{1}$, HAIWEI SANG ${ }^{1}$, \\ HAIJUN DONG ${ }^{2}$, YIFAN YANG ${ }^{1}$, CHANG MA $^{1}$ and TAO TAO ${ }^{1}$ \\ ${ }^{1}$ Department of Thoracic Surgery, The First Affiliated Hospital of Bengbu Medical College, Bengbu, Anhui 233030; \\ ${ }^{2}$ Department of Thoracic Surgery, Huzhou Central Hospital, Huzhou, Zhejiang 310000, P.R. China
}

Received July 23, 2020; Accepted March 11, 2021

DOI: $10.3892 / o r .2021 .8036$

\begin{abstract}
The present study aimed to investigate the role of partner of NOB1 homolog (PNO1) in esophageal cancer (EC). The expression levels of PNO1 in EC were primarily analyzed using data obtained from databases. PNO1 expression was also knocked down in EC cells (Eca-109 and TE1) to determine the biological effects of PNO1 on tumorigenesis in vitro and in vivo. In addition, possible downstream targets of PNO1 in EC were identified. The expression levels of PNO1 were upregulated in the tumor tissues compared with that noted in normal tissues. Moreover, the knockdown (KD) of PNO1 suppressed cell proliferation, migration and invasion, and promoted cell apoptosis $(\mathrm{P}<0.05)$. Furthermore, the protein expression levels of AKT1, Twist, Myc, mTOR, matrix metalloproteinase 2 (MMP2), nuclear factor (NF)- $\mathrm{B}$ p65 and $\beta$-catenin 1 (CTNNB1) were downregulated following the KD of PNO1 in Eca-109 cells $(\mathrm{P}<0.05)$. In addition, the overexpression of CTNNB1 reversed the effects of PNO1 KD in Eca-109 cells $(\mathrm{P}<0.05)$. In conclusion, the findings of the present study suggest that PNO1 promotes EC progression by regulating AKT1, Twist, Myc, mTOR, MMP2, NF- $\mathrm{kB}$ p65 and CTNNB1 expression.
\end{abstract}

\section{Introduction}

Esophageal cancer (EC) is the sixth leading cause of cancer-related mortality worldwide and the 5-year survival rate of patients is only $15-25 \%$ (1). Despite the significant advancements made in the treatment of EC, patients with EC have a poor prognosis, as EC cells can metastasize to lymph nodes, even at an early stage, and migrate to distant sites (2). Thus, possible biological targets for the treatment of EC require further investigation.

Correspondence to: Dr Tao Tao, Department of Thoracic Surgery, The First Affiliated Hospital of Bengbu Medical College, 287 Changhuai Road, Bengbu, Anhui 233030, P.R. China

E-mail: taotao02265@163.com

Key words: partner of NOB1 homolog, CTNNB1, esophageal cancer, AKT1, cell proliferation
Partner of NOB1 homolog (PNO1) is a highly conserved protein with a $\mathrm{K}$ homology $(\mathrm{KH})$ domain at its $\mathrm{C}$-terminal and two putative nuclear localization signals at its $\mathrm{N}$-terminal $(3,4)$. In mice, PNO1 was discovered to be involved in immune responses and proteasome activities (5). Currently, the oncogenic role of PNO1 in hepatocellular carcinoma and colorectal cancer has been determined $(6,7)$. However, the expression levels, biological effects and mechanisms of action of PNO1 in EC remain to be elucidated.

The present study first analyzed the expression levels of PNO1 in EC tissues using data obtained from The Cancer Genome Atlas (TCGA) database. Subsequently, the biological effects of PNO1 in EC were determined. Finally, potential downstream targets of PNO1 in EC were investigated.

\section{Materials and methods}

TCGA database. RNA-sequencing profiles of PNO1 expression in $162 \mathrm{EC}$ and 11 normal samples were downloaded from the TCGA database (https://tcga-data.nci.nih.gov/tcga). In addition, RNA-sequencing profiles of PNO1 expression in 653 normal samples were obtained from the Genotype-Tissue Expression (GTEx) database (http://xena.ucsc.edu), which is a database that provides information on normal samples from healthy participants. Both the 653 samples in GTEx database and 11 normal samples in TCGA database were used as normal samples $(8,9)$. The differentially expressed genes (DEGs) between control and cancerous samples were identified using the Limma package of R software (http://bioconductor. org/packages/release/bioc/html/limma.html) (10). The cut-off values for DEGs were $\left|\log ^{\text {fold change }}\right|>1$ and $\mathrm{P}<0.05$.

Cell lines and culture. EC cell lines (EC9706, Eca-109 and TE-1), the normal esophageal epithelial cell line HEEC, and the 293T cell line (Procell Life Technology Co., Ltd., Wuhan, China) were cultured in DMEM (HyClone; Cytiva) supplemented with $10 \%$ FBS (Gibco; Thermo Fisher Scientific, Inc.) at $37^{\circ} \mathrm{C}$ with $5 \% \mathrm{CO}_{2}$.

Construction of stable PNO1-knockdown (KD) and $\beta$-catenin (CTNNB1)-overexpressing cells. PNO1 was stably knocked down in Eca-109 and TE-1 cells using short hairpin RNA (sh), and CTNNB1 was stably overexpressed in Eca-109 cells only. sh-PNO1-KD, sh-PNO1-negative control (NC), sh-CTNNB1-OE and sh-CTNNB1-negative control (NC) 
were used for transfection. Lentiviral vectors were used for Eca-109 and TE-1 cell transfection as previously described (11). Images of the cells were obtained under a fluorescence microscope following transfection for $72 \mathrm{~h}$. Reverse transcription-quantitative PCR (RT-qPCR) and western blotting were used to analyze the transfection efficiency.

$R T-q P C R$. Total RNA was extracted and reversed transcribed into cDNA using TRIzol ${ }^{\circledR}$ reagent (Invitrogen; Thermo Fisher Scientific, Inc.) and Promega's Universal Riboclone cDNA synthesis system (Promega Corp.), respectively, according to the manufacturers' protocols. qPCR was subsequently performed using a SYBR Green Master mix (Takara Biotechnology Co., Ltd.), using GAPDH as the endogenous control. The following forward and reverse primers sequences were used for the qPCR: GAPDH forward, 5'-TGACTTCAA CAGCGACACCCA-3' and reverse, 5'-CACCCTGTTGCT GTAGCCAAA-3'; and PNO1 forward, 5'-TGTTAAACCCCT AAAGGGAGACC-3' and reverse, 5'-CCTTGTCCGTGTCAC ATTCTCT-3'. Expression levels were quantified using the $2^{-\Delta \Delta \mathrm{Cq}}$ method (12).

Western blotting. Total protein was extracted from cells using radioimmunoprecipitation lysis buffer (RIPA, Solarbio Technology Co., Beijing, China). The extracted protein was separated by SDS-PAGE and transferred onto PVDF membranes. The membranes were subsequently incubated with primary antibodies (Table I). Following the primary antibody incubation, the membranes were incubated with anti-mouse IgG (1:5,000; cat. no. sc-2005; Santa Cruz Biotechnology, Inc.) and anti-rabbit IgG (1:5,000; cat. no. sc-2004; Santa Cruz Biotechnology, Inc.) secondary antibodies. Protein bands were visualized using a Pierce ${ }^{\mathrm{TM}}$ ECL Western Blotting substrate (Thermo Fisher Scientific, Inc.).

Celigo cell counting assay. A Celigo cell counting assay was performed as previously described (13). Briefly, Eca-109 and TE-1 cells were seeded into 96-well plates at a density of $2 \times 10^{3}$ cells/well. Cells were cultured for a total of $120 \mathrm{~h}$, and cells were counted with a Celigo ${ }^{\circledR}$ Cell Imaging cytometer (Nexcelom Bioscience) every $24 \mathrm{~h}$.

Colony formation assay. A cell colony formation assay was performed as previously described (14). Briefly, Eca-109 and TE-1 cells were seeded into 6-well plates at a density of 800 cells/well. Following the culture of the cells for 2 weeks, cells were fixed with $1 \mathrm{ml}$ paraformaldehyde (4\%) for $40 \mathrm{~min}$ and stained with $1 \mathrm{ml}$ crystal violet dye solution $(0.1 \%)$ for $15 \mathrm{~min}$. Stained cells were visualized under a microscope (Olympus) and colonies with more than 10 cells were counted.

MTT assay. The MTT assay was performed as previously described (15). Briefly, Eca-109 and TE-1 cells were seeded into 96-well plates at a density of $2 \times 10^{3}$ cells/well and cultured for 5 days. Subsequently, $20 \mu 1$ MTT solution ( $5 \mathrm{mg} / \mathrm{ml}$; Gen-view Scientific, Inc.) was added into each well and incubated for $4 \mathrm{~h}$ at $37^{\circ} \mathrm{C}$. Following the incubation, $100 \mu \mathrm{l}$ DMSO was added to each well to dissolve the purple formazan crystals. The absorbance was measured at a wavelength of $490 \mathrm{~nm}$ to detect the optical density (OD) value.
Cell apoptosis assay. Cell apoptosis assay was performed using an Annexin V Apoptosis Detection kit (eBioscience; Thermo Fisher Scientific, Inc.) as previously described (16).

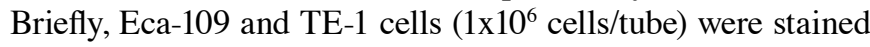
with $10 \mu \mathrm{l}$ Annexin $\mathrm{V}$-allophycocyanin, and apoptotic cells were analyzed by flow cytometry (FACSCalibur, Beckman Coulter).

Wound healing assay. The wound healing assay was performed as previously described (17) using a Celigo cytometer. Briefly, Eca-109 and TE-1 cells expressing GFP were seeded into 96-well plates at a density of $5 \times 10^{4}$ cells/well and a scratch was made in the cell monolayer. The fluorescence indicates the efficiency of transfection. After scratching, the cells were cultured in serum-free DMEM for $24 \mathrm{~h}$. Images of the scratch were obtained at 0 and $24 \mathrm{~h}$ using Celigo which can identify cells with green fluorescence and images were captured.

Cell Transwell assay. Cell Transwell assay was performed with Transwell kits (Corning, Inc.) as previously described (18). Briefly, $1 \times 10^{5}$ cells suspended in $100 \mu 1$ serum-free DMEM were seeded into the upper chambers. The lower chambers were filled with $600 \mu \mathrm{l}$ DMEM supplemented with $30 \%$ FBS. Following culture for $8 \mathrm{~h}$, cells were fixed with $4 \%$ paraformaldehyde for $30 \mathrm{~min}$ and stained with crystal violet aqueous solution (0.5\%). Cells were subsequently visualized under a microscope (Olympus).

Cell invasion assay. Cell invasion assay was performed using BioCoat ${ }^{\mathrm{TM}}$ Matrigel $^{\circledR}$ Invasion chambers (Corning, Inc.) as previously described (19). Briefly, $500 \mu \mathrm{l}$ serum-free medium was plated into both the upper and lower chambers for $2 \mathrm{~h}$ at $37^{\circ} \mathrm{C}$ to rehydrate the Matrigel matrix. Subsequently, $1 \times 10^{5}$ cells in $500 \mu \mathrm{l}$ serum-free DMEM were seeded into the upper chamber and $750 \mu 1$ DMEM supplemented with $30 \%$ FBS was added into the lower chamber. Following incubation for $8 \mathrm{~h}$, Giemsa staining solution was added, and images were captured using a microscope (Olympus).

Protein-protein interaction analysis. The BioGRID database (https://thebiogrid.org) (20) was used to identify proteins that interacted with PNO1 in humans.

Gene set enrichment analysis (GSEA). GSEA version 3.0 software (software.broadinstitute.org/gsea/index.jsp) was used for GSEA (21). A false discovery rate (FDR q-val) of $\leq 25 \%$ and nominal $\mathrm{P}<0.05$ were set as the cut-off values. The ggplot2 package (https://cran.r-project.org/web/packages/ggplot2/index. html) was used to merge the selected images.

Xenograft experiments. Twenty female BALB/c nude mice (age, 4 weeks, Shanghai Slake Experimental Animal Co., Ltd.) were subcutaneously inoculated with sh-PNO1-NC- or sh-PNO1-KD-transfected Eca-109 cells $\left(4 \times 10^{6}\right.$ cells suspended in $200 \mu \mathrm{l}$ PBS) to form tumors. All mice were euthanized by intraperitoneal injection of an overdose of $2 \%$ sodium pentobarbital $(100 \mathrm{mg} / \mathrm{kg})$, and the death was confirmed by cervical dislocation. The tumor volume of each mice was measured every three days for 14 consecutive days two weeks after inoculation using the following equation: 3.14/6 $\mathrm{x}$ (length $\mathrm{x}$ width $\mathrm{x}$ width). All animal experiments were conducted in accordance with 
Table I. List of the primary antibodies used in the western blot analysis.

\begin{tabular}{|c|c|c|c|c|c|c|}
\hline Gene & Abbreviation & Host & Company & Cat. no. & $\begin{array}{c}\text { Weight } \\
(\mathrm{kDa})\end{array}$ & Dilution \\
\hline Cadherin 1 & $\mathrm{CDH1}$ & Mouse & $\begin{array}{l}\text { Cell Signaling } \\
\text { Technology, } \\
\text { Inc. (CST) }\end{array}$ & $14472 \mathrm{~s}$ & 135 & $1: 100$ \\
\hline Mitogen-activated protein kinase 14 & P38 & Rabbit & CST & 8690 & 40 & $1: 300$ \\
\hline Phosphorylated-nuclear factor $\kappa \mathrm{B}$ & $p-N F K B$ & Rabbit & CST & $3033 \mathrm{~s}$ & 65 & $1: 200$ \\
\hline Matrix metallopeptidase 9 & $M M P 9$ & Rabbit & CST & $13667 \mathrm{~s}$ & 84 & $1: 300$ \\
\hline Mechanistic target of rapamycin kinase & $m T O R$ & Rabbit & CST & 2983 & 289 & $1: 300$ \\
\hline Catenin $\beta 1$ & $C T N N B 1$ & Rabbit & CST & 9562 & 92 & $1: 300$ \\
\hline Phosphorylated-AKT serine/threonine kinase & $p-A K T$ & Rabbit & CST & 4060 & 60 & $1: 1,000$ \\
\hline Cadherin 2 & $\mathrm{CDH} 2$ & Rabbit & CST & 13116 & 140 & $1: 100$ \\
\hline Phosphorylated-mitogen-activated protein kinase 14 & $p-P 38$ & Rabbit & CST & 4631 & 43 & $1: 300$ \\
\hline Phosphorylated-catenin $\beta 1$ & $p-C T N N B 1$ & Rabbit & CST & $2009 \mathrm{~s}$ & 92 & $1: 300$ \\
\hline Nuclear factor $\kappa \mathrm{B}$ p65 & $N F-\kappa B p 65$ & Rabbit & CST & 8242 & 65 & $1: 500$ \\
\hline MYC proto-oncogene, bHLH transcription factor & myc & Rabbit & Abcam & ab32072 & 57 & $1: 100$ \\
\hline NFKB inhibitor $\alpha$ & $N F K B I A$ & Rabbit & Abcam & $\mathrm{ab} 7217$ & 35.6 & $1: 300$ \\
\hline Matrix metallopeptidase 2 & $M M P 2$ & Rabbit & Abcam & ab37150 & 72 & $1: 300$ \\
\hline Twist family bHLH transcription factor & Twist & Rabbit & Abcam & ab50581 & 21 & $1: 300$ \\
\hline Fibronectin 1 & FN1 & Mouse & Abcam & ab6328 & $>250$ & $1: 100$ \\
\hline AKT serine/threonine kinase 1 & $A K T 1$ & Rabbit & Abcam & ab183758 & 56 & $1: 300$ \\
\hline Phosphorylated-mechanistic target of rapamycin kinase & $p-m T O R$ & Rabbit & Abcam & ab109268 & 289 & $1: 300$ \\
\hline Fartner of NOB1 homolog & PNO1 & Rabbit & $\begin{array}{l}\text { Santa Cruz } \\
\text { Biotechnology, } \\
\text { Inc. }\end{array}$ & sc-133263 & 31 & $1: 100$ \\
\hline Glyceraldehyde-3-phosphate dehydrogenase & $G A P D H$ & Mouse & $\begin{array}{l}\text { Santa Cruz } \\
\text { Biotechnology, } \\
\text { Inc. }\end{array}$ & sc-32233 & 36 & $1: 500$ \\
\hline
\end{tabular}

the Guide for the Care and Use of Laboratory Animals of the National Institutes of Health [National Research Council (US) Institute for Laboratory Animal Research, 1996] and were approved by the Ethics Committee of The First Affiliated Hospital of Bengbu Medical College.

Statistical analysis. Statistical analysis was performed using SPSS 22.0 software (IBM Corp.) and GraphPad Prism 7.0 software (GraphPad Software, Inc.). For western blot analysis, only one repeat was performed. However, for all other experiments, three repeats were conducted and experiments were further repeated if discordant results were obtained. Data are presented as the mean \pm SD. Statistical differences between groups were determined using unpaired Student's t-test or one-way ANOVA with Tukey test. The expression data of PNO1 mRNA in the 8 paired samples obtained from the TCGA database met the requirement for a parametric analysis, and paired Student's t-test was utilized. $\mathrm{P}<0.05$ was indicative of statistically significant differences.

\section{Results}

Expression levels of PNO1 in EC. To determine the expression levels of PNO1 in EC, PNO1 expression levels in $162 \mathrm{EC}$ and 664 normal samples from databases were analyzed. As shown in Fig. 1A, PNO1 expression levels were upregulated in the tumor tissues compared with that in the 664 normal tissues $(\mathrm{P}<0.0001)$. Paired tumor and non-tumor samples from the dataset were selected, and similar results were obtained $(\mathrm{P}=0.0364$; Fig. 1B). In addition, PNO1 mRNA expression levels in EC cell lines (EC9706, Eca-109 and TE-1) and the normal esophageal epithelial cell line, HEEC, were analyzed. As shown in Fig. 1C, PNO1 expression levels were upregulated in EC cells compared with that noted in the HEEC cells $(\mathrm{P}<0.0001)$. The PNO1 expression levels were highest in TE-1 cells, then Eca-109 cells, then EC9706 cells, and the lowest in HEEC cells. These data suggested that PNO1 mRNA expression levels may be upregulated in tumor samples compared with the levels in normal samples.

Successful establishment of stable PNO1 KD Eca-109 and $T E-1$ cells. To determine the function and mechanism of PNO1 in EC, PNO1 expression was knocked down in Eca-109 and TE-1 cells. The green fluorescence intensity was used to evaluate whether the virus-carried plasmid was successfully transfected into the cells. As shown in the fluorescence images in Fig. S1A and B, PNO1 expression was successfully knocked down in the Eca-109 and TE-1 cells, with a transduction 
A

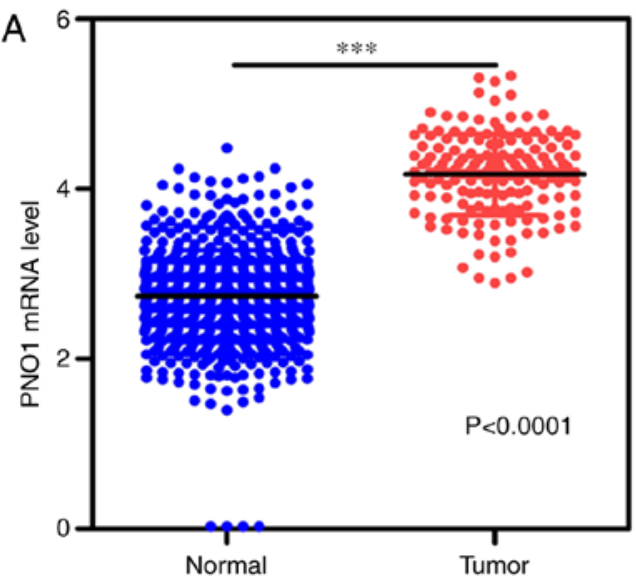

B

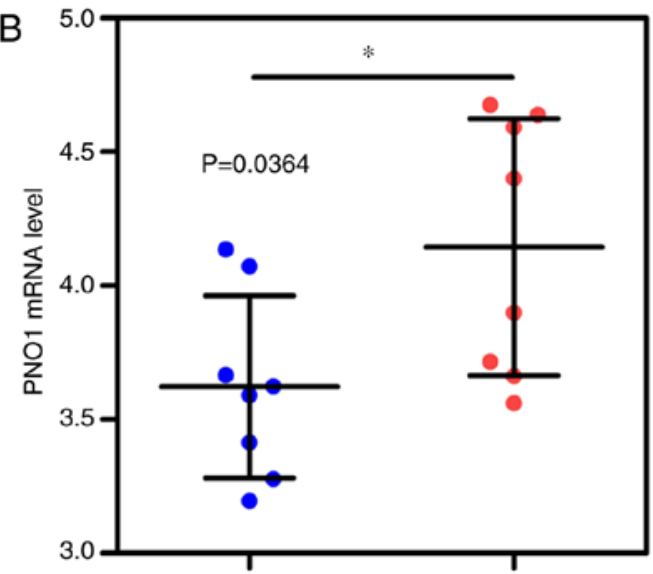

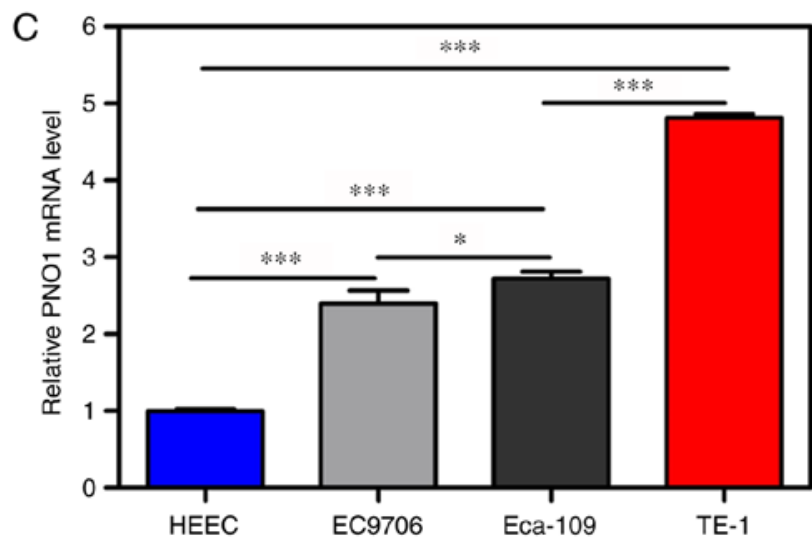

Figure 1. Expression levels of PNO1 in EC. (A) Data from the TCGA database indicated that PNO1 expression levels were upregulated in tumor tissue $(n=159)$ compared with normal tissues $(n=664)$. (B) Data from TCGA database indicated that PNO1 expression levels were upregulated in tumor tissues $(n=8)$ compared with normal tissues $(\mathrm{n}=8)$. (C) Reverse transcription-quantitative PCR analysis revealed that PNO1 expression levels were downregulated in normal cells (HECC) compared with tumor cell lines (TE-1, Eca-109 and EC9706). The data are presented as the mean \pm SD. "P<0.05, ${ }^{* * * *} \mathrm{P}<0.001$. EC, esophageal cancer; PNO1, partner of NOB1 homolog; TCGA, The Cancer Genome Atlas.

efficiency in both cells of $>80 \%$. In addition, PNO1 expression was successfully knocked down in Eca-109 and TE-1 cells, with transduction efficiencies of $>90$ and $70 \%$, respectively. Furthermore, RT-qPCR (Fig. S1C and D) and western blotting (Fig. S1E and F) were conducted to verify the transduction efficiency, and the results were consistent with the fluorescence microscopy results $(\mathrm{P}<0.0001)$. These results suggested that PNO1 expression was successfully silenced in both Eca-109 and TE-1 cells.

KD of PNO1 expression inhibits EC cell tumorigenesis in vitro and in vivo. To determine the effects of $\mathrm{PNO} 1$ on the proliferation of Eca-109 and TE-1 cells, Celigo cell counting, colony formation and MTT assays were performed. The results of the cell counting assay revealed that the cell amount/fold value was decreased by 3-fold in the sh-PNO1-KD-transfected cells compared with sh-PNO1-NC-transfected cells (Fig. 2A). Furthermore, the results of the colony formation assay demonstrated that the colony formation in sh-PNO1-KD-transfected Eca-109 cells was decreased by $\sim 15$-fold compared with sh-PNO1-NC-transfected Eca-109 cells, while the colony forming ability of sh-PNO1-KD-transfected TE-1 cells was 9-fold decreased compared with sh-PNO1-NC-transfected TE-1 cells (both $\mathrm{P}<0.0001$; Fig. 2B). In addition, the results of the MTT assay found that the OD490/fold value of
sh-PNO1-KD-transfected Eca-109 cells was decreased by 2-fold compared with sh-PNO1-NC-transfected Eca-109 cells, while the OD490/fold value of sh-PNO1-KD-transfected TE-1 cells was decreased by $\sim 2.5$-fold compared with sh-PNO1-NC-transfected TE-1 cells (Fig. 2C).

To determine the effects of PNO1 on the apoptosis of Eca-109 and TE-1 cells, the apoptotic rate of cells was investigated using flow cytometry. As shown in Fig. 2D, the apoptotic rate of sh-PNO1-KD-transfected Eca-109 cells was increased by 2-fold compared with sh-PNO1-NC-trasfected Eca-109 cells $(\mathrm{P}<0.0001)$, while that of sh-PNO1-KD-transfected TE-1 cells was increased by 5 -fold compared with sh-PNO1-NC-transfected TE-1 cells $(\mathrm{P}<0.0001)$.

To verify the effects of PNO1 on EC growth, in vivo xenograft experiments were performed and the tumor volume was calculated for $\sim 2$ weeks. As shown in Fig. 2E, the tumor volume was significantly decreased in the sh-PNO1-KD group compared with the sh-PNO1-NC group. Overall, these results suggested that the KD of PNO1 inhibited tumorigenesis in vitro and in vivo.

KD of PNO1 suppresses cell migration and invasion. To determine the effects of PNO1 on the migration and invasion of Eca-109 and TE-1 cells, wound healing, and Transwell migration and invasion assays were performed. The wound 

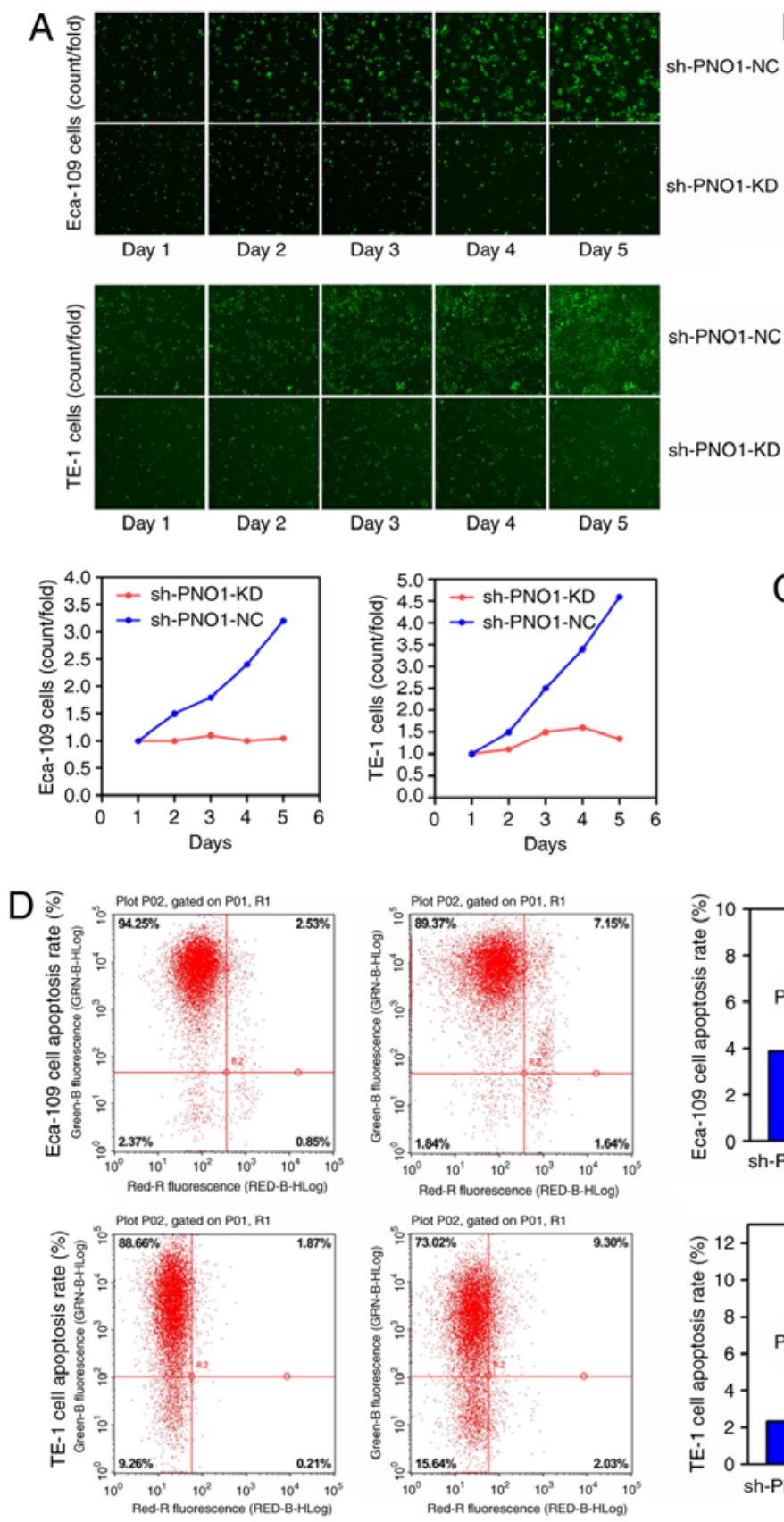

sh-PNO1-NC

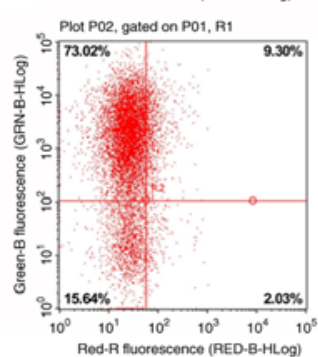

sh-PNO1-KD
B
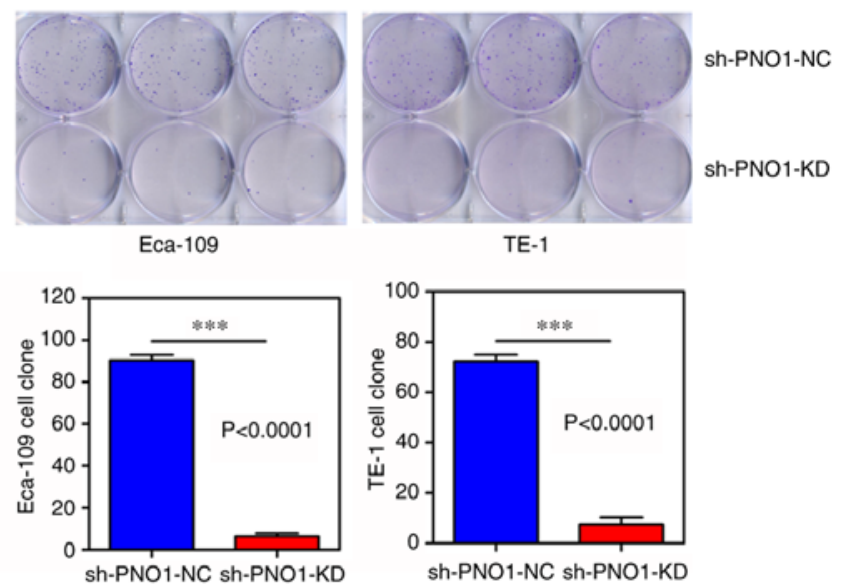

\section{C}
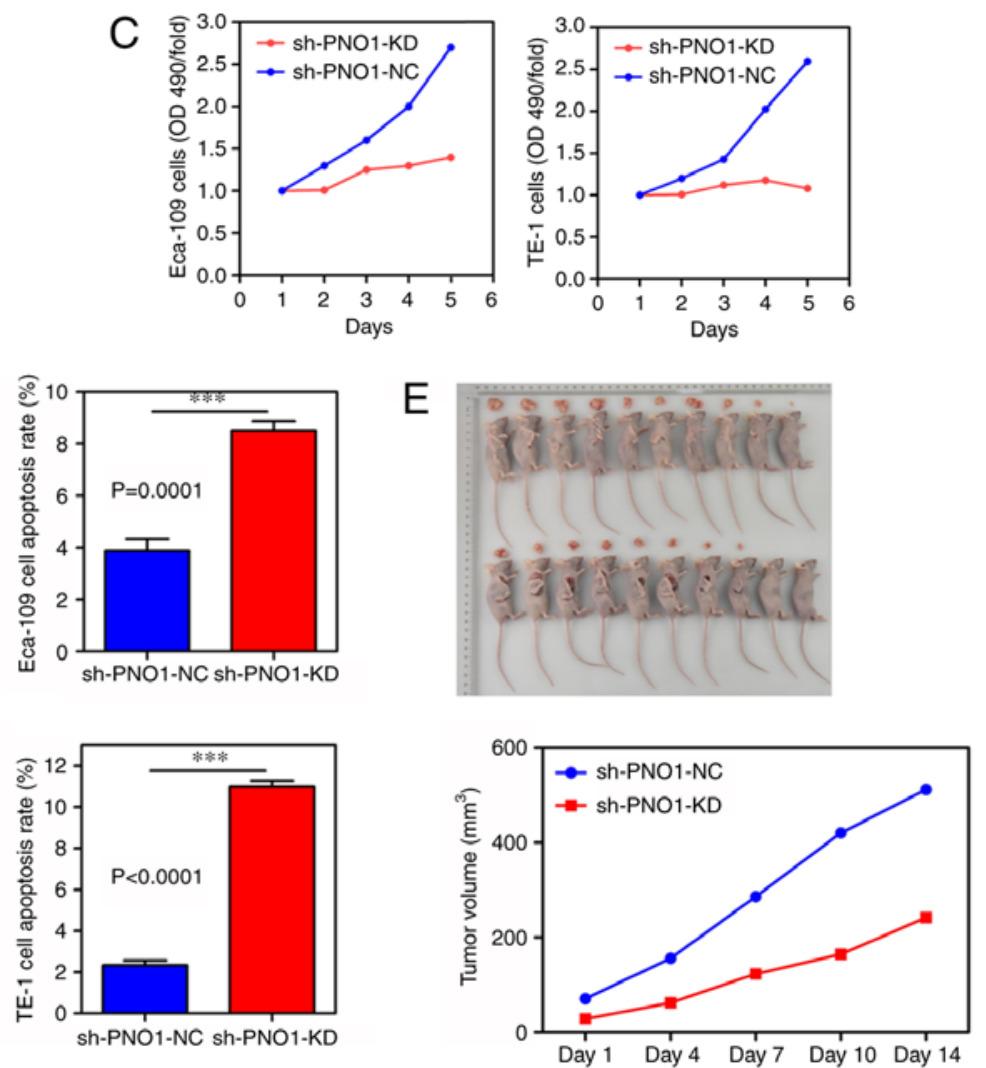

Figure 2. KD of PNO1 suppresses cell proliferation and promotes cell apoptosis. Results of the (A) Celigo cell counting assay, (B) colony formation assay and (C) MTT assay demonstrated that the cell proliferative ability was decreased following the KD of PNO1 in Eca-109 and TE-1 cells. (D) Results from flow cytometric analysis revealed that the cell apoptosis ability increased following the silencing of PNO1 in Eca-109 and TE-1 cells. (E) Tumor volume was decreased in the sh-PNO1 group compared with the sh-NC group. The data are presented as the mean \pm SD. ${ }^{* * *}$ P $<0.001$. PNO1, partner of NOB1 homolog; sh, short hairpin RNA; NC, negative control; KD, knockdown.

healing assay revealed that the migratory rates of the sh-PNO1-KD-transfected Eca-109 cells $(\mathrm{P}<0.0001)$ and sh-PNO1-KD-transfected TE-1 cells $(\mathrm{P}<0.001)$ were significantly decreased compared with sh-PNO1-NC-transfected Eca-109 and sh-PNO1-NC-transfected TE-1 cells (Fig. 3A). In particular, the migration rate of sh-PNO1-KD-transfected TE-1 cells was decreased by $\sim 12$-fold compared with sh-PNO1-NC-transfected TE-1 cells (Fig. 3A). Furthermore, the number of migratory sh-PNO1-KD-transfected Eca-109 and sh-PNO1-KD-transfected TE-1 cells was decreased compared with the sh-PNO1-NC-transfected Eca-109 and
sh-PNO1-NC-transfected TE-1 cells ( $\mathrm{P}<0.0001$; Fig. 3B). In more detail, the number of migratory sh-PNO1-KD-transfected Eca-109 cells was decreased by $\sim 10$-fold compared with the number of migratory sh-PNO1-NC-transfected Eca-109 cells, while the number of migratory sh-PNO1-KD-transfected TE-1 cells was decreased by $\sim 5$-fold compared with sh-PNO1-NC-transfected TE-1 cells. In addition, the number of invasive sh-PNO1-KD-transfected Eca-109 and sh-PNO1-KD-transfected TE-1 cells was significantly decreased compared with the number of sh-PNO1-NC-transfected Eca-109 and sh-PNO1-NC-transfected TE-1 cells. In 

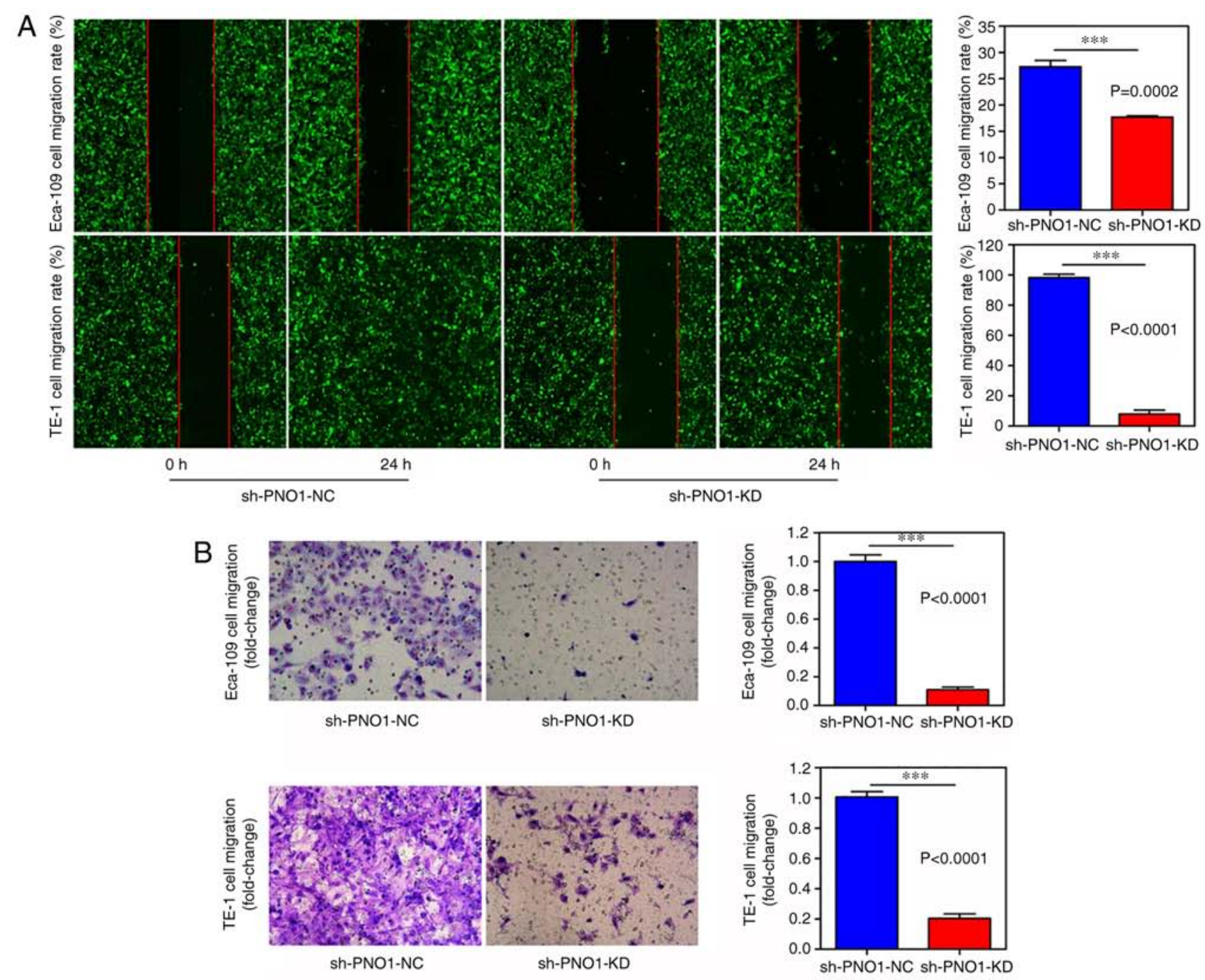

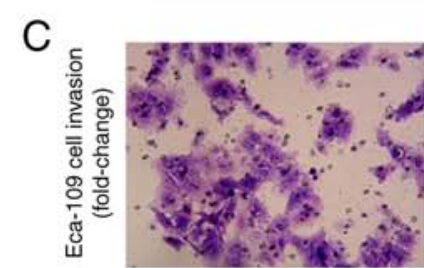

sh-PNO1-NC

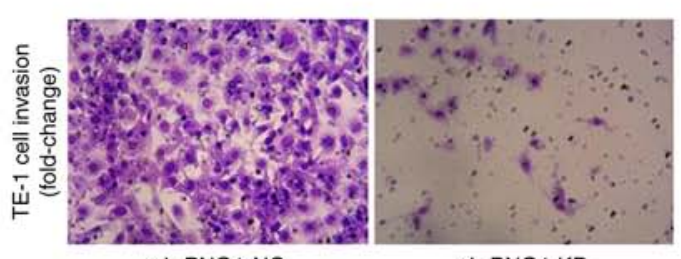

sh-PNO1-NC

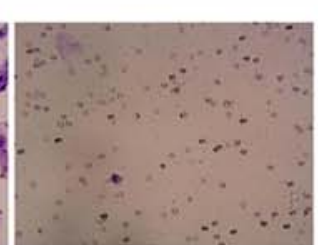

sh-PNO1-KD

sh-PNO1-KD
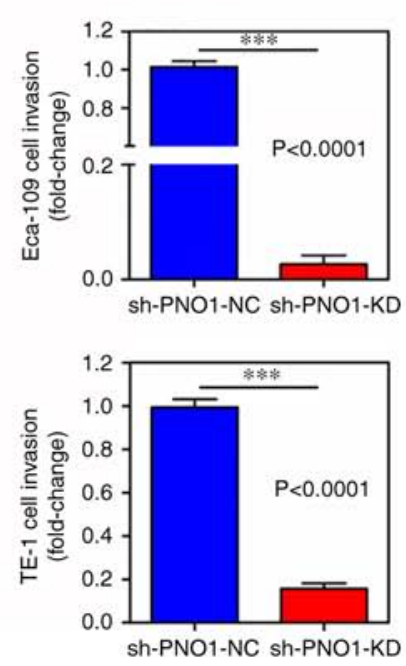

Figure 3. KD of PNO1 promotes cell migration and invasion. (A) Wound healing, (B) Transwell migration and (C) Transwell invasion assays demonstrated that the cell migration and invasion abilities were reduced following the knockdown of PNO1 in Eca-109 and TE-1 cells. The data are presented as the mean \pm SD. ${ }^{* * * *} \mathrm{P}<0.001$. PNO1, partner of NOB1 homolog; KD, knockdown.

fact, very few sh-PNO1-KD-transfected Eca-109 cells underwent invasion $(\mathrm{P}<0.0001$; Fig. $3 \mathrm{C})$.

Biological mechanisms of PNOI in EC. To determine the biological mechanisms of PNO1 in EC, GSEA was performed using data obtained from TCGA database. A total of 178 gene sets were enrolled for analysis, in which 21 and 25 gene sets met the criterion for the PNO1 high and low expression phenotypes, respectively. In samples with PNO1 high expression phenotypes, pathways related to the cell cycle and DNA replication 

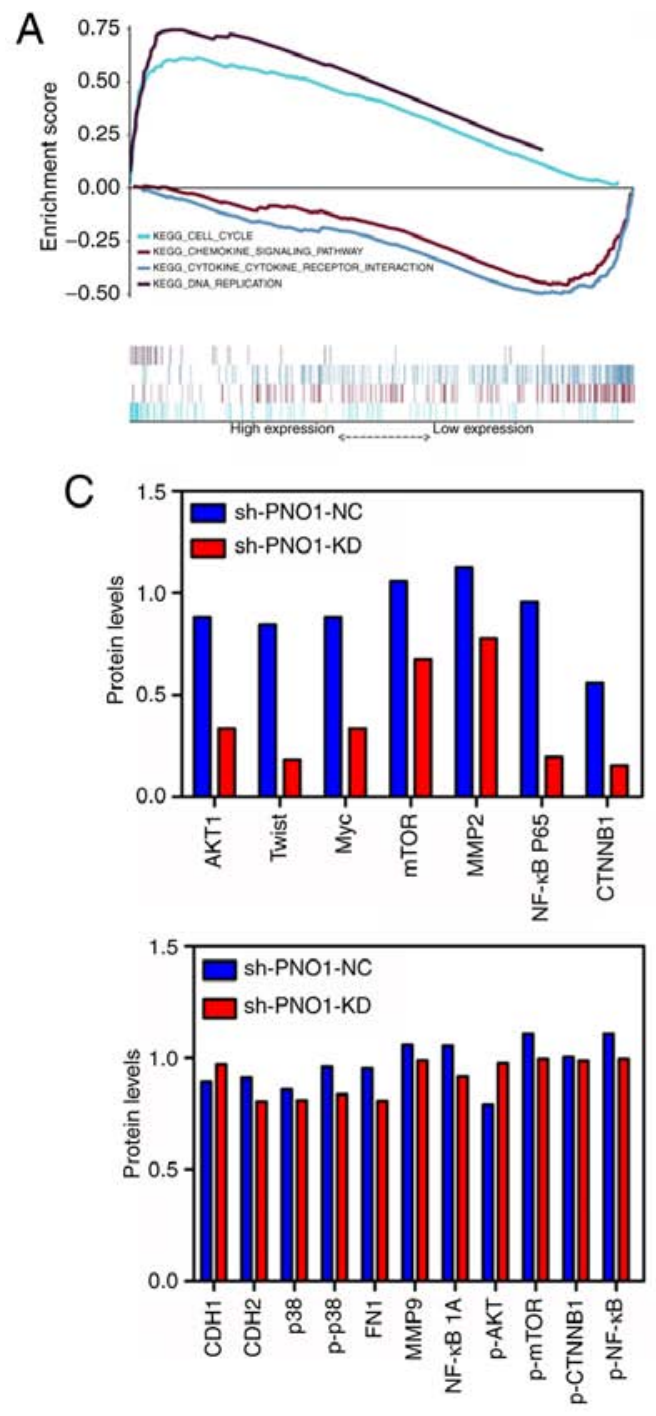

B

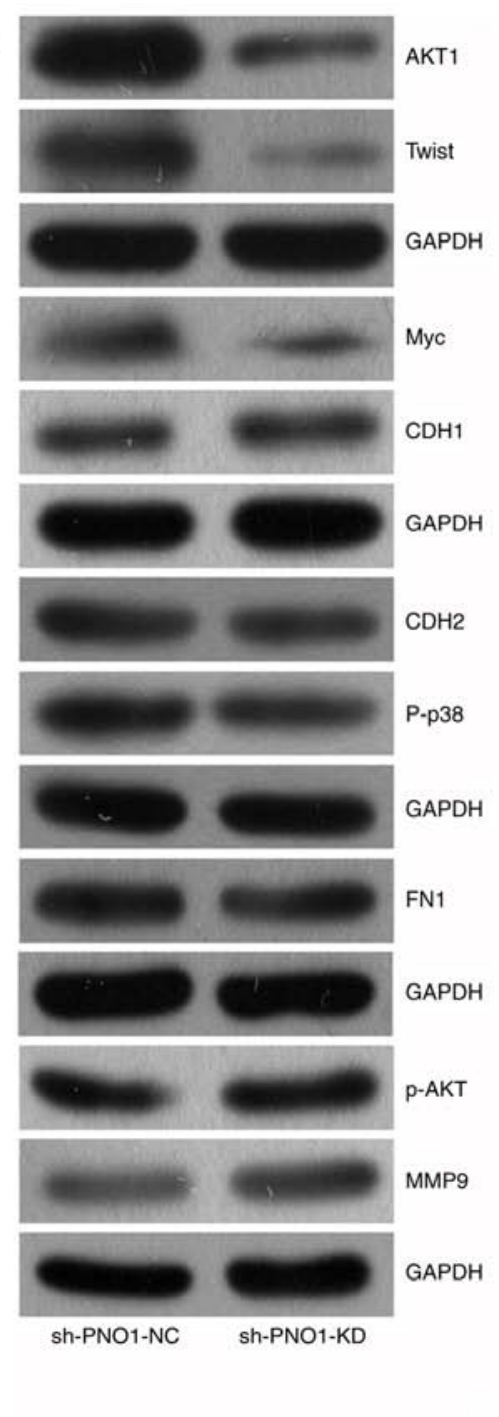

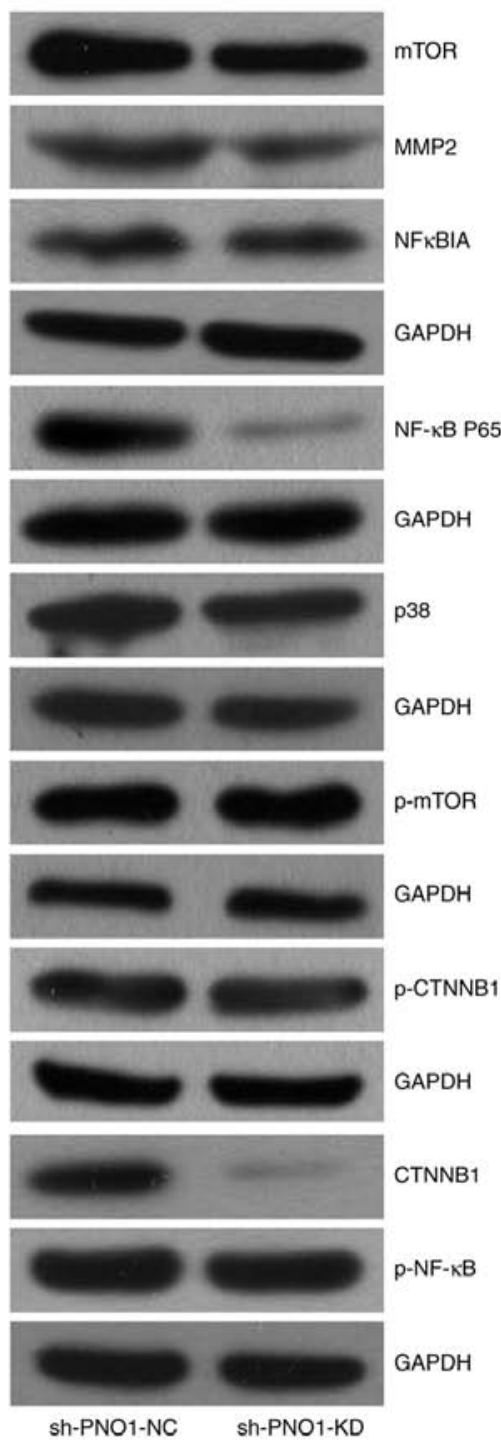

Figure 4. Biological mechanisms of PNO1 in esophageal cancer. (A) Gene Set Enrichment Analysis based on data from The Cancer Genome Atlas database found that pathways related to the cell cycle and DNA replication were upregulated in the PNO1 high expression phenotype, while pathways associated with chemokine signaling pathways and cytokine-cytokine receptor interaction were downregulated in the PNO1 low expression phenotype. (B and C) Protein expression levels of AKT1, Twist, Myc, mTOR, MMP2, NF-кB p65 and CTNNB1 were downregulated in sh-PNO1-KD-transfected Eca-109 cells compared with sh-PNO1-NC-transfected Eca-109 cells. Protein expression levels of CDH1, CDH2, p38, p-p38, FN1, MMP9, NFKBIA, p-AKT, p-mTOR, p-CTNNB1, p-NF-кB and Slug were similar in the sh-PNO1-KD-transfected Eca-109 and sh-PNO1-NC-transfected Eca-109 cells. CDH1, cadherin 1; p-, phosphorylated; FN1, fibronectin 1; MMP9, matrix metalloproteinase 9; NFKBIA, NF-кB inhibitor $\alpha$; PNO1, partner of NOB1 homolog; MMP2, matrix metalloproteinase 2; CTNNB1, $\beta$-catenin 1; KD, knockdown; sh, short hairpin RNA; NC, negative control.

were upregulated, while pathways associated with chemokine signaling pathways and cytokine-cytokine receptor interaction were downregulated in the PNO1 low expression phenotype (Fig. 4A), which indicated that PNO1 may promote EC growth via regulating genes related to the cell cycle and DNA replication. Thus, the expression levels of molecules related to the cell cycle were investigated in Eca-109 cells. As shown in Fig. 4B and C, the protein expression levels of AKT1, Twist, Myc, mTOR, matrix metalloproteinase (MMP)2, NF- $\kappa$ B p65 and CTNNB1 were downregulated in sh-PNO1-KD-transfected Eca-109 cells compared with in sh-PNO1-NC-transfected Eca-109 cells. However, the protein expression levels of cadherin $(\mathrm{CDH}) 1$, $\mathrm{CDH} 2$, p38, phosphorylated (p)-p38, fibronectin 1, MMP9,

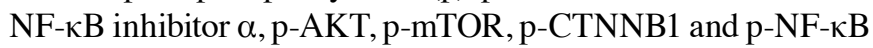
were similar in both sh-PNO1-KD-transfected Eca-109 and sh-PNO1-NC-transfected Eca-109 cells (Fig. 4B and C).
CTNNB1 may be a potential direct downstream target of PNO1 in EC. As shown in Fig. 4B and C, CTNNB1 was found to be regulated by PNO1. In addition, the results obtained from BioGRID identified that CTNNB1 interacted with PNO1 (data not shown). Hence, a rescue experiment was designed to verify the relationship between CTNNB1 and PNO1 in EC. We here overexpressed CTNNB1 in Eca-109 cells (Fig. 5A) and split Eca-109 cells into three groups: i) sh-PNO1-NC+sh-CTNNB1-NC-transfected Eca-109 cells; ii) sh-PNO1-KD+sh-CTNNB1-NC-transfected Eca-109 cells; and iii) sh-PNO1-KD + sh-CTNNB1-overexpression (OE)-transfected Eca-109 cells. Cell counting (Fig. 5B), MTT (Fig. 5C) and Transwell assays (Fig. 5D) were performed with the three groups. Compared with the sh-PNO1-NC+sh-CTNNB1-NC group, the proliferation and invasion of the sh-PNO1-KD+sh-CTNNB1-NC-transfected 

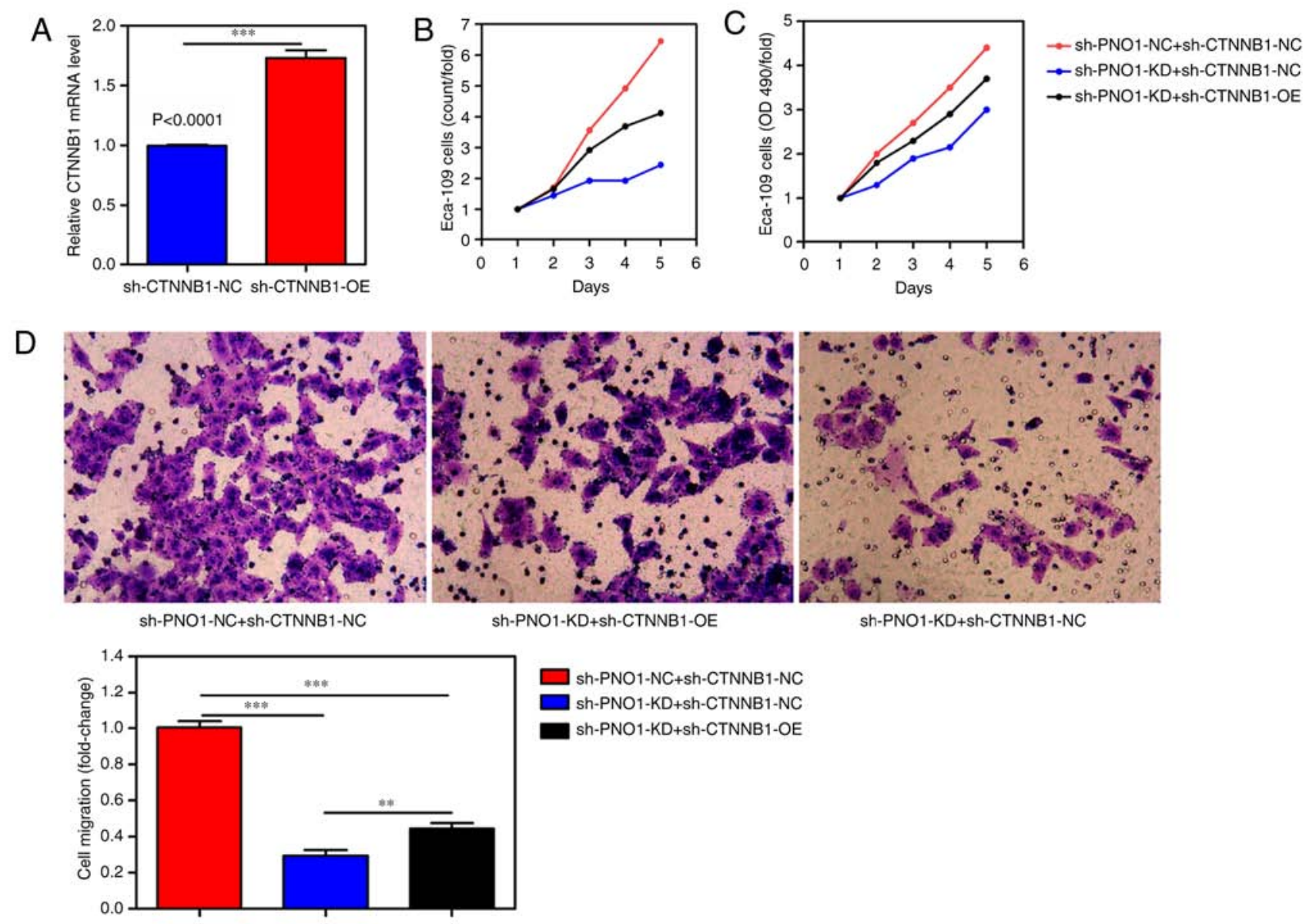

Figure 5. CTNNB1 is a potential direct downstream target of PNO1 in esophageal cancer. (A) CTNNB1 expression was overexpressed in the Eca-109 cells after transfection. Results of the (B) Celigo cell counting, (C) MTT, and (D) Transwell assays revealed that CTNNB1 overexpression abolished the effects of the knockdown of PNO1 in Eca-109 cells. The data are presented as the mean $\pm \mathrm{SD} .{ }^{* *} \mathrm{P}<0.01,{ }^{* * * *} \mathrm{P}<0.001$. CTNNB1, $\beta$-catenin 1; PNO1, partner of NOB1 homolog; KD, knockdown; sh, short hairpin RNA; NC, negative control.

Eca-109 cells was decreased. Conversely, compared with the sh-PNO1-KD+sh-CTNNB1-NC group, the proliferation and invasion of the sh-PNO1-KD+sh-CTNNB1-OE-transfected Eca-109 cells was increased. Altogether, these results suggested that the OE of CTNNB1 may abolish the effects of the KD of PNO1 in Eca-109 cells. Thus, CTNNB1 may be a potential direct downstream target of PNO1 in EC.

\section{Discussion}

Partner of NOB1 homolog (PNO1) is located on human chromosome 2q14 and consists of five introns and seven exons (4). The length of the full cDNA sequence of human PNO1 is 1,637 base pairs, which includes an open reading frame of 759 base pairs in length, and the weight of the PNO1 protein is $35 \mathrm{kDa}(3,4)$. In addition, PNO1 contains a $\mathrm{KH}$ domain, which is responsible for binding RNA and NIN1 (RPN12) binding protein 1 homolog (NOB1) at amino acids 157-230 in the human PNO1 C-terminal, and two nuclear localization signals at the PNO1 C-terminal from amino acids 23-29 and amino acids 53-58 (4). The biological function of PNO1 has been investigated over the past few years. In yeast, the interaction between PNO1 and NOB1 was found to be related to ribosome biogenesis $(5,7)$. The KD of PNO1 led to assembly defects of $26 \mathrm{~S}$ and $40 \mathrm{~S}$ ribosomal RNA (rRNA), decreased the levels of 18S rRNA and led to an accumulation of $32 \mathrm{~S}, 33 \mathrm{~S}$ and $35 \mathrm{~S}$ rRNA (7,22-24). In addition, previous studies reported that PNO1 was associated with the immune response and proteasome activities. These aforementioned findings indicated the potential crucial role of PNO1 in the physiological state. Furthermore, PNO1 has also been also identified as an oncogene in hepatocellular carcinoma and colorectal cancer $(5,7)$. However, to the best of our knowledge, the role of PNO1 in esophageal cancer (EC) remains unclear.

To investigate the role of PNO1 in EC, the present study analyzed the expression levels of PNO1 in EC tissues using data from TCGA database. Subsequently, the biological effects of PNO1 in EC were determined. Finally, potential downstream targets of PNO1 in Eca-109 cells were identified.

Shen et al analyzed microarray data performed with 14 pairs of colorectal cancer and corresponding tissues and preliminary identified PNO1, NUF2 component of NDC80 kinetochore complex, cell division cycle associated 5 and dachshund family transcription factor 1 as oncogenes in colorectal cancer (7). In addition, the study reported that PNO1 was a negative factor for predicting the overall survival of patients with colorectal cancer. The present study analyzed PNO1 expression levels in EC tissues from TCGA and GTEx databases and verified its expression in EC cells. The data demonstrated that PNO1 expression levels were upregulated in EC tissues compared with 
normal tissues. The differential expression of PNO1 indicated that PNO1 may play a role in EC progression. To verify this hypothesis, PNO1 expression was knocked down in Eca-109 and TE-1 cells, and the results revealed that the cell proliferation, migration and invasion abilities decreased, while the cell apoptosis ability was increased following the KD. In addition, in nude mice, a smaller tumor volume was observed following the KD of PNO1 expression. These results indicated that PNO1 may promote EC progression. Previous studies have also identified PNO1 as a tumor-promoting factor in hepatocellular carcinoma and colorectal cancer. For example, Dai et al reported that the growth and metastasis of hepatocellular carcinoma was inhibited following the silencing of PNO1 (6). Wang et al and Shen et al knocked down PNO1 expression in colorectal cell lines (PKO and HCT116) and found that the cell viability and colony formation rate were decreased and that the percentage of cells in the $\mathrm{G}_{0} / \mathrm{G}_{1}$ phase and undergoing apoptosis increased (5,7). These findings suggested that PNO1 may be an oncogene in EC.

As described above, PNO1 was identified as an oncogene in EC. Thus, the mechanism underlying the effects of PNO1 in EC was determined. Through GSEA, gene sets related to the cell cycle and DNA replication were found to be upregulated in the PNO1 high expression phenotype. These results indicated that PNO1 may promote EC growth via regulating genes related to the cell cycle and DNA replication. To verify this hypothesis, the expression levels of molecules related to the cell cycle were analyzed in Eca-109 cells with or without PNO1 KD. The NF- $\kappa \mathrm{B}$ and Wnt signaling pathways are involved in tumor proliferation, and NF- $\mathrm{NB}$ and CTNNB1 are key genes in the $\mathrm{NF}-\kappa \mathrm{B}$ and Wnt signaling pathways, respectively $(25,26)$. Thus, the expression levels of NF- $\kappa \mathrm{B}$ and CTNNB1 were analyzed following the KD of PNO1. The results revealed that the expression levels of both $\mathrm{NF}-\kappa \mathrm{B}$ and CTNNB1 were downregulated in the sh-PNO1-KD-transfected Eca-109 cells. In addition, the OE of CTNNB1 in sh-PNO1-KD-transfected Eca-109 cells reversed the decreased proliferation of Eca-109 cells. However, knockdown of PNO1 failed to change the level of phosphorylated CTNNB1. Two reasons may cause this phenomenon: Firstly, the level of phosphorylation is a process of modification of proteins after translation, and the level of total protein also represent its level before translation. So, CTNNB1 may be phosphorylated after translation. Secondly, protein level is regulated by upstream molecules, while phosphorylated protein is affected by kinases. Thus, it is possible that PNO1 affected the expression of some kinases and then the kinases regulated the phosphorylation of CTNNB1. Although, the level of phosphorylated CTNNB1 did not change, we can still draw a conclusion that CTNNB1 is a downstream target of PNO1 in EC.

PNO1 was observed to promote EC metastasis in previous studies, and the expression levels of Twist, Myc and MMP2, which are genes known to participate in the tumor metastasis process (27-29), were also analyzed following the KD of PNO1. The results suggested that PNO1 may promote EC metastasis via upregulating Twist, Myc and MMP2 expression levels. Altogether these data suggest that PNO1 may promote EC progression via upregulating NF- $\kappa \mathrm{B}$ p65, CTNNB1, Twist, Myc and MMP2 expression. Our study was consistent with previous studies (27-29).

There were limitations to the present research. Experiments to determine the direct association between PNO1 and
CTNNB1 were not performed. Also, we failed to detect the expression of PNO1 in fresh samples to verify the results obtained from public databases. Finally, we failed to detect the transfection efficiency of PNO1 in vivo.

In conclusion, the findings of the present study suggested that PNO1 may promote EC progression by regulating the expression of AKT1, Twist, Myc, mTOR, MMP2, NF- $\mathrm{B}$ p65 and CTNNB1.

\section{Acknowledgements}

Not applicable.

\section{Funding}

The Natural Science Key Projects of Bengbu Medical College (no. BYKY2019087ZD) funded this research.

\section{Availability of data and materials}

The datasets used during the present study are available from the corresponding author upon reasonable request.

\section{Authors' contributions}

TT and GW both made substantial contributions to the conception and design of this study. GW, QL, CL, GD, HS, HD, YY and $\mathrm{CM}$ made substantial contributions to the analysis and interpretation of the data. TT and GW both made substantial contributions to writing the manuscript. All authors approved the final version to be published and are accountable for all aspects of the work.

\section{Ethics approval and consent to participate}

The present study was approved by the Ethics Committee of the First Affiliated Hospital of Bengbu Medical College (Bengbu, Anhui, China).

\section{Patient consent for publication}

Not applicable.

\section{Competing interests}

The authors declare that they have no competing interests.

\section{References}

1. Domper Arnal MJ, Ferrández Arenas Á and Lanas Arbeloa Á: Esophageal cancer: Risk factors, screening and endoscopic treatment in western and eastern countries. World J Gastroenterol 21: 7933-7943, 2015.

2. Udagawa $\mathrm{H}$ and Akiyama H: Surgical treatment of esophageal cancer: Tokyo experience of the three-field technique. Dis Esophagus 14: 110-114, 2001.

3. Gibson TJ, Thompson JD and Heringa J: The KH domain occurs in a diverse set of RNA-binding proteins that include the antiterminator NusA and is probably involved in binding to nucleic acid. FEBS Lett 324: 361-366, 1993.

4. Zhou GJ, Zhang Y, Wang J, Guo JH, Ni J, Zhong ZM, Wang LQ, Dang YJ, Dai JF and Yu L: Cloning and characterization of a novel human RNA binding protein gene PNO1. DNA Seq 15: 219-224, 2004. 
5. Wang $\mathrm{X}, \mathrm{Wu} \mathrm{T}, \mathrm{Hu} \mathrm{Y}$, Marcinkiewicz M, Qi S, Valderrama-Carvajal $\mathrm{H}$, Luo $\mathrm{H}$ and $\mathrm{Wu}$ J: Pnol tissue-specific expression and its functions related to the immune responses and proteasome activities. PLoS One 7: e46093, 2012.

6. Dai H, Zhang S, Ma R and Pan L: Celecoxib inhibits hepatocellular carcinoma cell growth and migration by targeting PNO1. Med Sci Monit 25: 7351-7360, 2019.

7. Shen A, Chen Y, Liu L, Huang Y, Chen H, Qi F, Lin J, Shen Z, $\mathrm{Wu} \mathrm{X}, \mathrm{Wu} \mathrm{M}$, et al: EBF1-mediated upregulation of ribosome assembly factor PNO1 contributes to cancer progression by negatively regulating the p53 signaling pathway. Cancer Res 79: 2257-2270, 2019.

8. Gao L, Li X, Nie X, Guo Q, Liu Q, Qi Y, Liu J and Lin B: Integrated analysis of lymphocyte infiltration-associated lncRNA for ovarian cancer via TCGA, GTEx and GEO datasets PeerJ 8: e8961, 2020.

9. Zhang X, Wen X, Feng N, Chen A, Yao S, Ding X and Zhang L: Increased expression of T-box transcription factor protein 21 (TBX21) in skin cutaneous melanoma predicts better prognosis: A study based on the cancer genome atlas (TCGA) and genotype-tissue expression (GTEx) databases. Med Sci Monit 26: e923087, 2020.

10. Ritchie ME, Phipson B, Wu D, Hu Y, Law CW, Shi W and Smyth GK: limma powers differential expression analyses for RNA-sequencing and microarray studies. Nucleic Acids Res 43 e47, 2015 .

11. Fang KP, Dai W, Ren YH, Xu YC, Zhang SM and Qian YB: Both Talin-1 and Talin-2 correlate with malignancy potential of the human hepatocellular carcinoma MHCC-97 L cell. BMC Cancer 16: 45, 2016

12. Livak KJ and Schmittgen TD: Analysis of relative gene expression data using real-time quantitative PCR and the 2(-Delta Delta C(T)) method. Methods 25: 402-408, 2001.

13. Vinci M, Gowan S, Boxall F, Patterson L, Zimmerman M, Court W, Lomas C, Mendiola M, Hardisson D and Eccles SA: Advances in establishment and analysis of three-dimensional tumor spheroid-based functional assays for target validation and drug evaluation. BMC Biol 10: 29, 2012.

14. Sun R, Wu J, Chen Y, Lu M, Zhang S, Lu D and Li Y: Down regulation of Thrombospondin 2 predicts poor prognosis in patients with gastric cancer. Mol Cancer 13: 225, 2014.

15. Moodley S, Koorbanally NA, Moodley T, Ramjugernath D and Pillay M: The 3-(4,5-dimethylthiazol-2-yl)-2,5-diphenyl tetrazolium bromide (MTT) assay is a rapid, cheap, screening test for the in vitro anti-tuberculous activity of chalcones. J Microbiol Methods 104: 72-78, 2014.

16. Fadok VA, Voelker DR, Campbell PA, Cohen JJ, Bratton DL and Henson PM: Exposure of phosphatidylserine on the surface of apoptotic lymphocytes triggers specific recognition and removal by macrophages. J Immunol 148: 2207-2216, 1992

17. Arsic N, Bendris N, Peter M, Begon-Pescia C, Rebouissou C, Gadéa G, Bouquier N, Bibeau F, Lemmers B and Blanchard JM: A novel function for cyclin A2: Control of cell invasion via RhoA signaling. J Cell Biol 196: 147-162, 2012.
18. Song Y, Dong MM and Yang HF: Effects of RNA interference targeting four different genes on the growth and proliferation of nasopharyngeal carcinoma CNE-2Z cells. Cancer Gene Ther 18: 297-304, 2011

19. Zengel P, Ramp D, Mack B, Zahler S, Berghaus A, Muehlenweg B, Gires $\mathrm{O}$ and Schmitz S: Multimodal therapy for synergic inhibition of tumour cell invasion and tumour-induced angiogenesis. BMC Cancer 10: 92, 2010.

20. Chatr-Aryamontri A, Oughtred R, Boucher L, Rust J, Chang C, Kolas NK, O'Donnell L, Oster S, Theesfeld C, Sellam A, et al: The BioGRID interaction database: 2017 update. Nucleic Acids Res 45D: D369-D379, 2017.

21. Subramanian A, Tamayo P, Mootha VK, Mukherjee S, Ebert BL Gillette MA, Paulovich A, Pomeroy SL, Golub TR, Lander ES and Mesirov JP: Gene set enrichment analysis: A knowledge-based approach for interpreting genome-wide expression profiles. Proc Natl Acad Sci USA 102: 15545-15550, 2015.

22. Senapin S, Clark-Walker GD, Chen XJ, Séraphin B and Daugeron MC: RRP20, a component of the 90S preribosome, is required for pre-18S rRNA processing in Saccharomyces cerevisiae. Nucleic Acids Res 31: 2524-2533, 2003.

23. Tone Y and Toh-EA: Noblp is required for biogenesis of the 26S proteasome and degraded upon its maturation in Saccharomyces cerevisiae. Genes Dev 16: 3142-3157, 2002.

24. Vanrobays E, Leplus A, Osheim YN, Beyer AL, Wacheul L and Lafontaine DL: TOR regulates the subcellular distribution of DIM2, a KH domain protein required for cotranscriptional ribosome assembly and pre-40S ribosome export. RNA 14: 2061-2073, 2008.

25. Dolcet X, Llobet D, Pallares J and Matias-Guiu X: NF- $\kappa B$ in development and progression of human cancer. Virchows Arch 446: 475-482, 2005.

26. Tanabe S, Aoyagi K, Yokozaki H and Sasaki H: Regulation of CTNNB1 signaling in gastric cancer and stem cells. World J Gastrointest Oncol 8: 592-598, 2016.

27. Khan MA, Chen HC, Zhang D and Fu J: Twist: A molecular target in cancer therapeutics. Tumour Biol 34: 2497-2506, 2013.

28. Wang XN, Su XX, Cheng SQ, Sun ZY, Huang ZS and Qu TM: MYC modulators in cancer: A patent review. Expert Opin Ther Pat 29: 353-367, 2019.

29. Wang HL, Zhou PY, Zhang Y and Liu P: Relationships between abnormal MMP2 expression and prognosis in gastric cancer: A meta-analysis of cohort studies. Cancer Biother Radiopharm 29: $166-172,2014$.

This work is licensed under a Creative Commons Attribution-NonCommercial-NoDerivatives 4.0 International (CC BY-NC-ND 4.0) License. 